\title{
Probing turbulence in OMC1 at the star forming scale: observations and simulations $\dagger$
}

\author{
Maiken Gustafsson ${ }^{1} \ddagger$, Axel Brandenburg ${ }^{2}$, \\ Jean-Louis Lemaire ${ }^{3}$ and David Field ${ }^{1}$ \\ ${ }^{1}$ Department of Physics and Astronomy, University of Aarhus, 8000 Aarhus C, Denmark \\ email: maikeng@phys.au.dk, dfield@phys.au.dk \\ ${ }^{2}$ NORDITA, Blegdamsvej 17, 2100 Copenhagen $\emptyset$, Denmark \\ ${ }^{3}$ Observatoire de Paris \& Université de Cergy-Pontoise, LERMA \& UMR 8112 du CNRS, \\ 92195 Meudon, France
}

\begin{abstract}
Using radial velocities of vibrationally excited $\mathrm{H}_{2}$ emission in $\mathrm{OMC} 1$ we present the structure functions and the scaling of the structure functions with their order at scales ranging from $70 \mathrm{AU}$ to $30000 \mathrm{AU}$ extending earlier related studies to scales lower by two orders of magnitude. The structure functions for OMC1 show clear deviations from power laws at $1500 \mathrm{AU}$. The scaling of the higher order structure functions with order deviates from predicted theoretical scalings. Observational results are compared with simulations of supersonic hydrodynamic turbulence. The unusual scaling is explained as a selection effect of preferentially observing the shocked part of the gas. The simulations are unable to reproduce the deviations from power laws of the structure functions.
\end{abstract}

Keywords. turbulence, shock waves, ISM: kinematics and dynamics, ISM: molecules, ISM: jets and outflows

\section{Introduction}

The energy spectrum and the related structure functions have been observed to follow power laws in many molecular clouds over a broad range of scales (e.g. Heyer \& Brunt (2004)). This feature is usually explained as a manifestation of an energy cascade in a turbulence dominated medium, resulting in self-similar structures without any characteristic scales. However, energy may be injected into or removed from the system by processes such as self-gravity, star formation and supernova explosions and may thus introduce breaks in the energy cascade. In order to understand the evolution of molecular clouds and the importance of turbulence in the star formation process it is essential to identify the presence of these energy modifying processes and the scales at which they occur. This can be achieved by analyzing structure functions of the velocity and should involve a close interplay between observations and numerical simulations. Here we use high spatial resolution $\left(0.15^{\prime \prime}\right)$ infrared observations of velocities in the massive star forming region OMC1. We then compare the observational results with numerical simulations of supersonic hydrodynamic turbulence.

$\dagger$ Based on observations obtained at the Canada-France-Hawaii Telescope (CFHT) which is operated by the National Research Council of Canada, the Institut National des Sciences de l'Univers of the Centre National de la Recherche Scientifique of France, and the University of Hawaii.

$\ddagger$ Present address: Max-Planck-Institute for Astronomy, Königstuhl 17, 69117 Heidelberg, Germany 

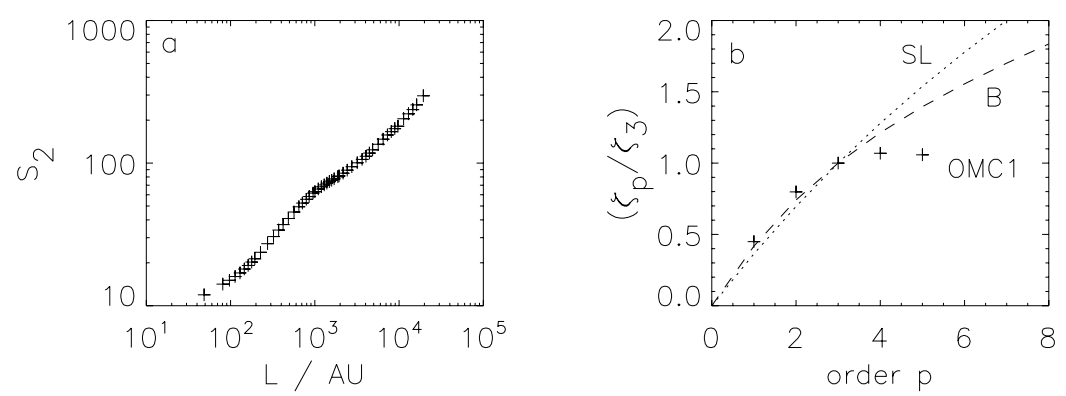

Figure 1. a) Second order structure function of radial velocities in OMC1. b) Scaling exponents of structure functions of order $p$ normalized to $\zeta_{3}$ versus order. For comparison are shown the theoretical scaling of She-Leveque (SL) and Boldyrev(B).

\section{Structure functions in OMC1}

Near infrared K-band observations of the strongest $\mathrm{H}_{2}$ emission line in Orion, $\mathrm{v}=1$ $0 \mathrm{~S}(1)$ at $2.121 \mu \mathrm{m}$, are used as a tracer of radial gas velocities in the BN-KL region of OMC1. The data were obtained at the CFHT with a Fabry-Perot interferometer in conjunction with adaptive optics (the GriF instrument) by spectral scanning of the $\mathrm{H}_{2}$ line (Gustafsson et al. (2003); Nissen et al. (2006)). Data consist of a field of $89^{\prime \prime} \times$ $67^{\prime \prime}$ or $41 \cdot 10^{3} \times 31 \cdot 10^{3} \mathrm{AU}$ (assuming a distance to OMC1 of $460 \mathrm{pc}$, (Bally et al. (2000)) centered on the BN object. The AO system achieved a spatial resolution of $0.15^{\prime \prime}(70 \mathrm{AU})$. The radial velocity at each spatial position was derived by spectral line fitting to the line profiles provided by the Fabry-Perot and relative velocities are determined with an accuracy of between $1 \mathrm{~km} \mathrm{~s}^{-1}(3 \sigma)$ in the brightest regions and $8-9 \mathrm{~km} \mathrm{~s}^{-1}$ in the weakest regions (Gustafsson et al. (2006a)). The emission of excited $\mathrm{H}_{2}$ observed here traces hot, dense gas, where excitation occurs primarily through shocks. Thus the cold gas goes unobserved.

Using the radial velocity map of OMC1 we obtain the structure functions

$$
S_{p}(L)=\left\langle B(\vec{r}) B(\vec{r}-\vec{\tau})|v(\vec{r})-v(\vec{r}-\vec{\tau})|^{p}\right\rangle=\left\langle|\Delta v|^{p}\right\rangle .
$$

Here $v$ is the line of sight velocity and the average is extended over all spatial positions $\vec{r}$ and all lags $\vec{\tau}$ where $L=|\vec{\tau}| . B(\vec{r})$ is the brightness at position $\vec{r}$. We thus weight each velocity difference by the product of the brightness of the two spatial positions involved, thereby giving more weight to the brightest regions which exhibit the highest accuracy in the radial velocity. Using brightness weighting assures that the result is less influenced by noise than if no weighting is used (Gustafsson et al. (2006a)). For homogeneous, isotropic turbulence the structure functions are known to follow power laws, $S_{p}(L) \sim L^{\zeta_{p}}$, in the inertial range, $\eta \ll r \ll L$, where $\eta$ is the dissipation scale and $L$ is the integral scale. The scaling exponents, $\zeta_{p}$, are expected to be characteristic of the turbulence involved e.g. incompressible or compressible - and universal for all scales, L.

The second order structure function derived from the OMC1 data is shown in Fig. 1a. At lags larger than $2000 \mathrm{AU}$, that is, over scales varying about an order of magnitude, the structure function is well represented by a power law. However, there is a clear deviation from from a power law at $1500 \mathrm{AU}$ associated with an excess of energy at this scale and below. This indicates a characteristic scale.

For structure functions of order 1 to 5 we use the concept of extended self-similarity introduced by Benzi et al. (1993) to obtain power law fits and the normalized scaling 
all
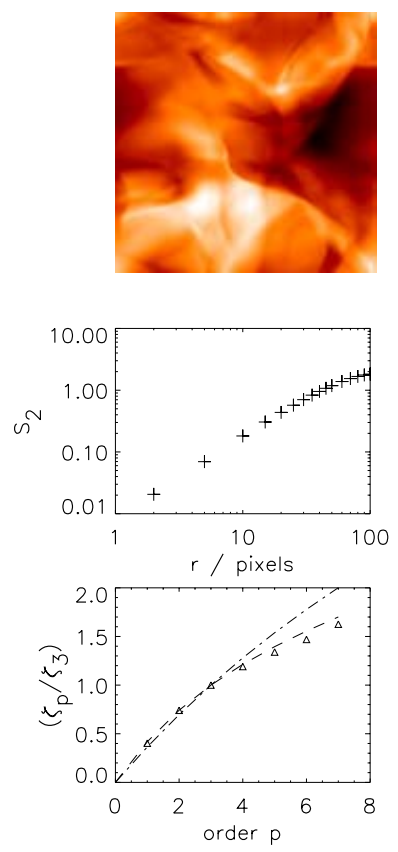

all shocks
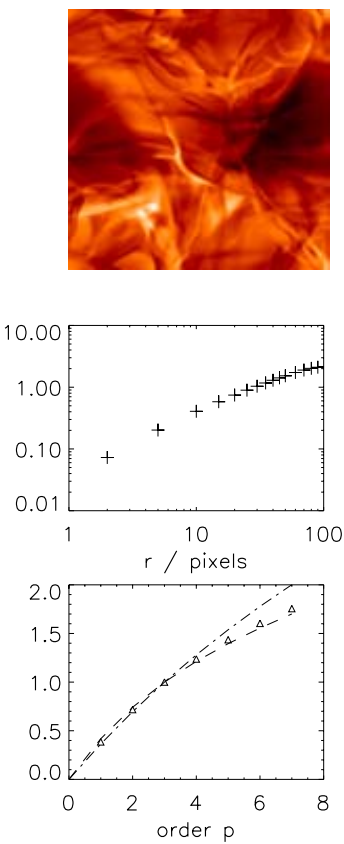

strong shocks
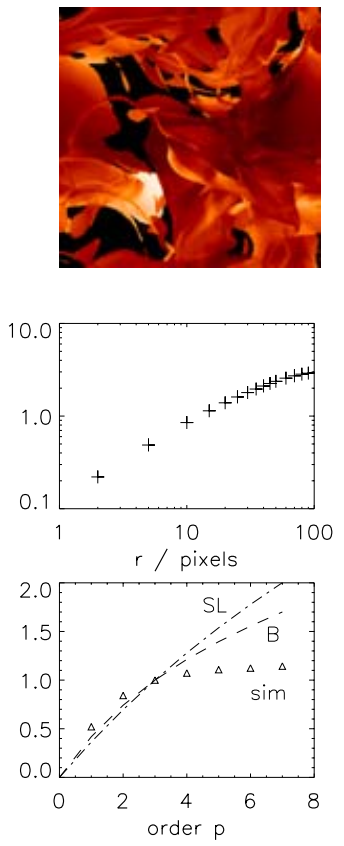

Figure 2. Results for projected maps including all points in the simulation (1st column) and for subsets including all shocks $(\nabla \cdot \vec{u}<0,2$ nd column $)$ and only strong shocks $(\nabla \cdot \vec{u}<-25$, 3rd column). Top row: projected radial velocity maps. Second row: second order structure functions. Third row: scaling exponents of the structure functions compared to the She-Leveque and Boldyrev scaling.

exponents $\zeta_{p} / \zeta_{3}$. These are plotted in Fig. $1 \mathrm{~b}$ as a function of order and are compared with the scaling exponents predicted by the She \& Leveque (1994) model of incompressible turbulence and the model of Boldyrev (2002) for supersonic, compressible turbulence. The scaling exponents derived from the velocity in OMC1 are seen to deviate from both the She-Leveque and the Boldyrev scaling at $p \geqslant 4$. The OMC1 scaling exponents show signs of becoming constant at $\zeta_{p} / \zeta_{3} \sim 1$ or even slightly decreasing for $p>4$, in contrast to the theoretical scalings, which are monotonically increasing.

\section{Numerical simulations}

In order to understand some of the features found in OMC1 we use data from numerical simulations of supersonic isothermal compressible turbulence. The simulations are purely hydrodynamic and are forced on random scales (Gustafsson et al. (2006b)). The root mean square Mach number is 3 in the simulation presented here. The viscosity is enhanced in the neighbourhood of shocks thereby broadening the physical size of shocks. The hydrodynamic equations are solved on a 3 -dimensional periodic mesh of size $256 \times 256 \times$ 256 using the PENCIL CODE (http://www.nordita.dk/software/pencil-code).

Since the main aim is to test if we can reproduce the features found in the observational data we want the numerical data to resemble best the physical properties of the observations. First, the observed radial velocity at each spatial position represents a projected velocity along the line-of-sight. Accordingly we project the numerical 3D velocity components onto a $2 \mathrm{D}$ map of only radial velocity. The projected radial velocity 
is found by density weighed averaging. Second, we observe a subset of the gas in OMC1 consisting largely of shocked gas. In the simulations we extract regions where shocks occur by selecting regions with strong negative velocity divergence, $\nabla \cdot \vec{u}<\mathrm{C}$. Here $\mathrm{C}$ is a negative cut-off value and only simulated points satisfying $\nabla \cdot \vec{u}<\mathrm{C}$ are included in the calculation of the projected radial velocity.

Figure 2 shows maps of projected radial velocities, the second order structure functions calculated from those maps and related scaling exponents. The maps shown correspond to including all points $(C=\infty)$, including all shocks $(C=0)$ and including only stronger shocks $(C=-25)$. The structure functions, represented by the 2 nd order structure function in Fig. 2, for all values of $\mathrm{C}$ are well approximated by power laws. This is in contrast to the structure functions of radial velocities found in OMC1 (Fig. 1) which showed clear deviations from power laws. This suggests that the deviations are not due to the fact that we observe only shocked gas, but arise from physical processes local to the OMC1. It is interesting to note that the preferred scale of $1500 \mathrm{AU}$ detected in the velocities in OMC1 is similar to the size of low-mass protostellar outflows which could in fact re-inject energy into an otherwise turbulent region. If a large number of such outflows are present they could act as a local driving agent for the turbulence and create a bump in the energy cascade similar to that found in OMC1.

The scaling exponents of the structure functions follow the relation predicted by Boldyrev (2002) for supersonic turbulence for the maps including all points and all shocked regions. However, for the map where only strong shocks are included the scaling exponents deviate from the theoretical scalings and resemble the scaling found in OMC1 (Fig. 1). Thus the unusual scaling observed in OMC1 can be explained as an effect of observing only the hot, shocked gas.

\section{Conclusion}

We have shown that the velocity field of OMC1 is not self-similar but contains a preferred scale size of $1500 \mathrm{AU}$ associated with an excess of energy. This cannot be reproduced by hydrodynamical simulations and more advanced simulations are necessary.

\section{Acknowledgements}

MG and DF acknowledge the support of the Instrument Center for Danish Astrophysics (IDA) and the Aarhus Center for Atomic Physics (ACAP) and the natural science funding council (FNU). The Danish Center for Scientific Computing is acknowledged for granting time on the Horseshoe cluster in Odense.

\section{References}

Bally, J., O'Dell, C.R. \& McCaughrean, M.J. 2000, AJ 119, 2919

Benzi, R., Ciliberto, S., Tripiccione, R., Baudet, C., Massaioli, F. \& Succi, S. 1993 Phys. Rev. E 48,29

Boldyrev, S. 2002, ApJ 569, 841

Gustafsson, M., Kristensen, L.E., Clénet, Y., Field, D., Lemaire, J.L., Pineau des Forêts, G., Rouan, D. \& Le Coarer, E. 2003, A\&A 411, 437

Gustafsson, M., Field, D., Lemaire, J.L. \& Pijpers, F.P. 2006, A\&A 445, 601

Gustafsson, M., Brandenburg, A., Lemaire, J.L. \& Field, D. 2006, A $\mathscr{A} A$ 454, 815

Heyer, M.H \& Brunt, C.M. 2004, ApJ 615, L45

Nissen, H.D., Gustafsson, M., Lemaire, J.L., Clenet, Y., Rouan, D. \& Field, D. 2006, astro$\mathrm{ph} / 0511226$

She, Z. \& Leveque, E. 1994, Phys. Rev. Lett. 72, 336 


\section{Discussion}

MAC LOw: OMC-1 is a coherent Hubble outflow, not a region of uniform turbulence, so application of statistical models drawn from uniform turbulence is questionable. A model of an expanding, fragmenting shell is more appropriate (e.g., Xu, Stone \& Mundy, or McCaughrean \& Mac Low).

Gustafsson: That may be true and yet the statistical models applied have to reproduce some of the observed features. The Fabry-Perot data indicate that the $\mathrm{H}_{2}$ emission in OMC1 is somewhat more complicated than a Hubble outflow and could suggest that the outflow have triggered turbulence that we now see.

Burton: $1500 \mathrm{AU}$ at Orion is $\sim 3$ arcsec, which is the typical scale size of the $\mathrm{H}_{2^{-}}$ emitting $\mathrm{HH}$ knots in OMC-1. These are not the sites of star formation in themselves. The knots are part os a Hubble-type expanding flow resulting from an impulsive event $\sim 500$ years ago.

Gustafsson: A scale of $\sim 1500 \mathrm{AU}$ is indeed the size of some of the $\mathrm{H}_{2}$ emitting clumps in $\mathrm{OMC} 1$, and we also see that clearly from the brightness data. That does not necessarily imply that radial velocities are coherent on the same scale. But they are! One more thing is that the Fabry Perot data clearly show that all of these clumps observed cannot be part of a Hubble-type flow with a common origin. 